\title{
National project "Labor productivity" as a factor in the development of the non-resource sector of the economy
}

\author{
Alexander Pobedin ${ }^{1}$, and Sergey Ezhov ${ }^{2, *}$ \\ ${ }^{1}$ Head of the Department of Economics and Management, Ural Institute of Management - a branch of \\ the Russian Academy of National Economy and Public Administration under the President of the \\ Russian Federation, Yekaterinburg, Russia \\ ${ }^{2}$ Leading Specialist of the Joint Stock Company “Zlatoust Machine-Building Plant”, Zlatoust, Russia
}

\begin{abstract}
The article examines the role of labor productivity in the sustainable development of the economy and considers the main goals of ensuring its growth. The main problems are identified, the solution of which allows the implementation of the National Project "Labor Productivity". So, thanks to participation in this project, business entities of the non-resource sector of the economy are introducing programs aimed at identifying and reducing costs that do not affect the final product. Thus, a new worldview is being formed, aimed at continuous implementation of changes in order to reduce costs. The most important thing is that thanks to these actions, it is possible to improve the production process and increase the profit for a business entity. In our opinion, lean manufacturing should better explain the specific forms of work situations that allow you to adapt to modes of operation other than standard ones. Therefore, in the course of future activities, it is necessary to develop various scenarios for getting out of the current situations, the data about which are unknown at the time of project development. This will ultimately have a positive impact on the development of the economy at the municipal, regional and federal levels.
\end{abstract}

\section{Introduction}

Improving the quality of life of citizens of the Russian Federation and disclosing their internal potential is impossible without significant progress in increasing innovation in industry and the application of new methods and technologies in management. Of particular relevance is the solution to the problem of reducing labor productivity, which, according to research by the analytical center under the Government of the Russian Federation in 20052015 , formed dangerous prerequisites for: a drop in the quality of products manufactured in the state; significant lag of the Russian economy from developed countries. Only in 2017, according to the analysis carried out by the All-Russian Research Institute of Labor of the Ministry of Labor of Russia, there was a slight increase in labor productivity. The reasons that negatively affect labor productivity are the physical and moral obsolescence of

* Corresponding author: egov_s@mail.ru 
equipment, tools, tooling and technologies used in enterprises of the real sector of the Russian economy, and the gap between the competence of employees of organizations and the requirements of modern society for innovation and modernization processes.

Unfortunately, until recently, the activities implemented to increase labor productivity were local and limited in time. To change the current situation, the national project "Labor Productivity" (formerly "Increasing Labor Productivity and Supporting Employment"), which has been implemented in the territory of the Russian Federation since 2019, is intended.

The technology for increasing labor productivity within the framework of the National Project is the concept of lean production. This is due, first of all, to its high level of efficiency and profitability when introduced into the production process from the receipt of an application from the client to the shipment of finished products to the warehouse or to the customer's address.

Since 2019, Zlatoust Machine-Building Plant JSC, the largest machine-building enterprise in the Chelyabinsk region in terms of the volume of state defense orders, has become a participant in the national project "Labor Productivity", whose project for the implementation of lean manufacturing was recognized as the best in the country.

\section{Methodology}

In our research, we applied several scientific methods. First of all, this is an analysis of the existing literature on lean manufacturing, since any project is based on existing knowledge and must fill in the gaps in it. Analysis of the literature on lean manufacturing aims to summarize and combine existing publications on this topic.

In addition, we analyzed the state policy carried out within the framework of the National Project "Labor Productivity" and broadcast through the ANO "Federal Center of Competence".

Among the research methods, we also applied case studies. The case was the production of Mechta and Zlata electric stoves by Zlatoust Machine-Building Plant JSC, the largest machine-building enterprise in terms of the state defense order in the Chelyabinsk Region. The case method allowed us to study in depth a specific case of application of the concept of lean production in the framework of the enterprise's participation in the National Project "Labor Productivity".

In the process of applying the case method, we analyzed and processed data on JSC "Zlatmash", the production of household appliances, the National Project "Labor Productivity" and the ANO "Federal Center of Competence".

When considering the case, we identified the advantages of introducing the concept of lean production for the enterprise, but also noted the negative consequences for the team. We have proposed an option that takes into account the needs of Zlatmash and the operators who manufacture the Mechta and Zlata electric stoves.

\section{Results}

Globalization and the emergence of new technologies influenced the management models that had to be adopted by companies. Indeed, in order for organizations to develop and maintain their competitiveness in an increasingly changing environment, it is necessary to improve the adopted management model [1]. In response to this implementation of Lean Management has attracted a large number of companies operating in various sectors, as this approach improves competitiveness, productivity and quality. [2]. 
Lean Manufacturing was developed in the Japanese automotive sector as a management strategy aimed at continuous improvement, end-user value creation and waste elimination [3], a people-centered approach with the ultimate goal of creating more and more value. with less time, space and fewer mistakes [4].

Taiichi Ohno (1988), founder of lean manufacturing at Toyota, identifies three key issues of concept effectiveness: Muda - eliminating waste, Mura - analyzing and controlling variability in demand, and Muri - eliminating overloading of equipment and employees [5]. Lean Management provides companies with the tools to survive in a global marketplace that demands the best quality, the fastest, and the lowest prices in the amount needed to maintain a company's profitability [1].

Over time, Lean has evolved to become an interdisciplinary subject related to several topics such as operations management, supply chain, organizational behavior [6], human resource management [7], and strategic management [8].

The concept itself is based on the regulation of consumer demand for products by constantly improving the quality of manufactured products and the competence of personnel, reducing stocks and work in progress, as well as redistributing the load between operators (from overloaded to unloaded, as well as combining operations in order to free operators) [9].

The main methods of the concept of "lean manufacturing" include:

1) Elimination of hidden losses - identification and elimination of operations that do not affect the creation of products.

2) $5 \mathrm{~S}$ system - decomposition of production issues, improving the ergonomics of the operator's workspace and production culture.

3) Operational changeover (SWED) - reducing the loss of time for equipment readjustment aimed at increasing the volume of product manufacturing, reducing work in progress and finished products in warehouses [10].

4) Eliminate the possibility of making mistakes [11].

5) The Just in Time (JIT) system is the manufacture of exactly those types of products that the customer needs, provided that the enterprise fulfills the specified parameters - time and volume.

6) "Value stream map" is aimed at structuring the movement of resources required in the production process.

7) "Kaizen" is necessary for the uninterrupted improvement of processes (production and non-production) occurring in a business entity, and is aimed at optimizing consumed resources [12].

It is the concept of lean production that became the basis of the National Project "Labor Productivity", implemented in the territory of the Russian Federation since 2019 and designed to improve the economic performance of economic entities of the country. Indeed, according to the data of the Organization for Economic Cooperation and Development, labor productivity in 2017 returned to the level of 2014 and amounted to $\$ 26.5$ / hour, which is $7 \%$ higher than in 2016, but significantly lower than the level (more than 100\%) than in other OECD member states [13].

The decline in labor productivity in Russia has a number of specific features:

1) Slowing down of the process of declining industrial activity in Russian regions due to the imbalance in the sectors of the economy.

2) A small percentage of enterprises belonging to the sectors of the knowledge economy and the digital economy.

3) The lack of clarity on the motives of the state when pursuing a policy of supporting domestic enterprises.

4) Lack of desire of the organization's personnel (top management and workers) to implement breakthrough technologies in the production process. 
5) Low level of installation and commissioning of modern equipment to replace the old one.

6) A significant degree of monopolization of the economy and an insignificant degree of competition.

7) Minimization of the personnel's interest in the growth of labor productivity.

8) Natural migration of the population to regions with a high level of wages.

9) "Personnel hunger".

10) The discrepancy between the desires of citizens and the needs of the labor market.

11) Low adaptation of citizens to changes in the labor market.

The reasons for the low labor productivity in the regions are:

1) Insufficient attention of the leaders of the regions of the Russian Federation to the problem of increasing labor productivity and the lack of appropriate practical actions.

2) The low level of managerial and technological competencies of enterprise managers, which is necessary for a qualitative leap in labor productivity.

3) Underdeveloped financial mechanisms necessary for the implementation of projects to improve production efficiency.

4) High social risks of mass layoffs and ineffective mechanisms for the rapid search for new jobs, retraining and advanced training of employees.

5) Lack and low productivity of human capital as a leading factor in the intellectual and economic development of the Russian Federation and its regions due to low investment in it.

That is why the National Project is focused on:

1) Increase in labor productivity at Russian enterprises.

2) Increase in the share of enterprises supplying products for export.

3) Carrying out measures to reduce bureaucratic barriers for enterprises.

The objectives of this project are:

1) Reduction of regulatory and administrative restrictions that impede the growth of labor productivity.

2) Stimulating the introduction of advanced management, organizational and technological solutions to increase labor productivity and modernize fixed assets, including through the provision of tax preferences.

3) Formation of a personnel training system aimed at teaching the basics of increasing labor productivity.

4) Creation of a system of methodological and organizational support for increasing labor productivity at enterprises (Passport of the national project "Labor productivity and employment support").

It is assumed that by 2024, 10,000 business entities from 85 regions of the Russian Federation will participate in the National Project. So in 2019, a number of 36 subjects became participants in the program, and in 2020 - another 14. However, labor productivity growth is largely a matter of technology, and in the context of sanctions and a pandemic, Russia's technological openness remains questionable [14].

The implementation of these tasks involves:

1) Theoretical and practical training of 200,000 managers of Russian enterprises (top and middle management);

2) Providing grant support to the best enterprises implementing lean manufacturing;

3) Changing the concept of the formation of personnel policy in organizations.

Created on December 18, 2017 by the decision of the Presidium of the Council under the President of the Russian Federation for Strategic Development and Priority Projects, the Federal Center for Competence in the Sphere of Labor Productivity (hereinafter - FCC) is the operator of the National Project in terms of targeted support of enterprises.

FCC implements programs at large and medium-sized business entities focused on identifying and reducing costs that do not affect the final product, as well as training experts 
in lean manufacturing at the enterprise, transmitting their knowledge to personnel. In the long term, this will give a "domino effect" for the implementation of lean manufacturing in all divisions of the organization.

Targeted grants will be directed to organizations implementing innovative and rationalization proposals to reduce costs and increase labor productivity. Thus, a new outlook of the personnel of enterprises is being formed, aimed at the continuous implementation of changes in order to reduce costs [15]. The procedure for diagnosing the production system for the need for investment injections is as follows:

1) A business entity identifies the need for investment support and analyzes the possibility of attracting its own funds (redistributing available resources), after which it submits an application for certification of the FCC.

2) FCC experts within 18 days conduct an initial assessment of the application, analyzing the company's cash flow, after which they issue a certificate.

3) A business entity submits an application for investment support to the Entrepreneurship Development Fund (hereinafter - EDF).

4) The EDF experts analyze the application within 60 working days, carry out a comprehensive examination and make a decision on the allocation of investment support at the expert council.

5) In case of a positive decision on the allocation of funds, a bilateral agreement is signed between the business entity and the EDF on the issuance of a loan.

6) EDF monitors the growth of labor productivity - in the first year of the investment loan $-5 \%$, in the last - at least $20 \%$.

The measures to support enterprises in the non-resource sector of the economy also include tax incentives and concessional lending from IDF. In order for an organization to be eligible to become a participant in the program, it must have the following parameters: the volume of annual revenue - from 400,000,000 rubles. up to $80,000,000,000$ rubles; participation of foreign capital - no more than $25 \%$ of the authorized capital. One example of the effectiveness of the program is that in $69 \%$ of organizations participating in the National Project at the end of 2019-beginning of 2020, labor productivity growth was more than $10 \%$ [16].

Consider the results of the implementation of the national project "Labor productivity" on the example of JSC "Zlatmash". In December 2019, the enterprise became one of the participants in the National Project, and in two years the working group on the implementation of changes has achieved significant results.

In order to optimize the production process, project working groups created for each site develop a production analysis system, analyze the time to solve problems and their degree of importance.

There was a reduction in staff movements. For example, in the past, oven installation and frame assembly were performed remotely from the main assembly line. Now this distance is 15 meters, that is, now workers walk 3000 meters less per shift. They were able to achieve such results at the assembly site due to the compactness of production.

There was also a division of areas of responsibility. Previously, the installation of the control unit took place in one area for both tabletop and floorboards. Now this operation for stationary models is performed in a specially designated area - on separate tables for assembly. Thanks to the changes, the wasted time for reconfiguring the installation disappeared, as a result, the workers forgot about the problem of tangled wires. Accordingly, labor productivity has increased.

The division of areas of responsibility also occurred between the repair and operational personnel for the inspection and maintenance of equipment. For this, the frequency and optimal routes of bypasses and inspections were determined, as well as key points of the equipment according to the analysis of breakdown statistics. 
The members of the working group created a "supermarket" and installed shelving units. They contain the minimum sets of components, tools and hardware that allow performing operations assigned to the performer. The operator will simply do his job, while completely eliminating unnecessary movements around the shop.

In addition, the operations of riveting the brackets and installing heating elements on the oven were combined. Also, at one workplace, the frame is assembled on the tilter and the thermal insulation is installed. Thus, the number of jobs has been reduced from eight to four, and the number of employed workers - from six to four, due to the reduction of losses and the compact placement of jobs. The freed employees were reassigned to other operations. In addition, improvements have been made to other assembly lines.

In order to increase the motivation of employees during the project implementation period, communication channels are also being developed. For example, the system "Effective meetings at information stands" is being implemented both at individual sites and at the level of the entire production and technical complex. In this way, employees become aware of the changes taking place in the company and begin to strive to achieve performance in their workplaces.

The next stage in the implementation of the National Project program at the enterprise was the creation of a project office. The management of the enterprise set a clear task of building a strategy for the phased implementation of projects for the development of production in all areas of the enterprise in order to achieve the set goal - to increase productivity.

The next project was the "Optimization of the assembly of tabletop plates" project, which resulted in an increase in plate production, a decrease in work in progress by 2,620 pieces $(25 \%)$, an increase in output and average wages by $19 \%$.

A natural continuation of the activities of the working group and the project office was the project "Optimization of the welding and coating process". During the implementation of the project, the members of the working group determined the throughput of the equipment: after making measurements and decomposing the budgetary indicators of production into components, they calculated the percentage of rejects, and identified areas with a higher percentage.

Based on the results of the production analysis, bottlenecks were identified. For example, in case of poor-quality surface degreasing, which leads to a problem during painting, the operator is forced to repeat this procedure, otherwise the enamel on the poorly degreased surface will curl up or turn into "bumps", as a result of which the production plan will not be fulfilled. In addition, after rebuilding the hangers and increasing the number of operators, the problem of kiln throughput will be solved.

A production analysis system has also been developed, which will be conducted on a daily basis at the site and identify problems affecting the production process for their further analysis and elimination.

In the welding area, operators spend a lot of time moving from place to place and changeovers. After implementing the changes (organizing a "supermarket" and moving welding machines), the total time savings will amount to 2,728 hours per year (124 shifts). In addition, due to a $74.5 \%$ reduction in travel and changeover time by $54 \%$, throughput increased by $10 \%$. Also, two new resistance welding machines and eight resistance welding distributors were purchased.

At the dry coating site, due to the restoration of the dry enameling line and the purchase of spare parts (including containers and the organization of a "supermarket"), there will be an increase in output, a decrease in the process time by $25 \%$ and consumables by $30 \%$, and rejects will also be reduced - from $9.4 \%$ to $4 \%$. 


\section{Discussion}

As we can see, the introduction of lean production is beneficial from an economic point of view for JSC "Zlatmash". However, in our opinion, further application of the concept at the sites for the production of electrical household stoves will bring the opposite effect. The reductions in unnecessary movement offered by Lean manufacturing can lead to poor operator health.

Since unnecessary movements do not affect the value stream, the Lean manager will force you to transform the necessary movements into reflexes. Since any increase in productivity implies a reduction in time, human activity is reduced to the use of force, and the operator will get tired of the monotony of work over time, which will affect production.

We propose to distinguish, depending on the circumstances, whether the elimination or reduction of movements is a resource or an obstacle for the operator. Because it really says whether movement can be a useful resource for his health (regulation of muscle tension, recovery time, etc.), but also for its effectiveness (time to reflect on the task at hand, helping a colleague). It also involves considering work as the interaction of the physical, psychological (cognitive, mental) and social components of the operators.

Lean, in its quest for overall company efficiency, does not know how to reward the subjective component by which all operators contribute in their own way to what the organization expects of it. This is the reason why Lean Manufacturing lacks conceptual guidelines to convert perceived waste into a substantial resource.

Observation of a gesture or movement is only meaningful if it can be interpreted with the intentions of its author in mind. In the future, it is necessary to ensure, on the one hand, the health, well-being, safety and development of operators, and on the other hand, the quality, reliability and efficiency of their activities. Otherwise, the level of performance achieved so far will drop and return to its original value.

\section{Conclusion}

The concept of "lean manufacturing" effectively improves business processes. This tool can be used in almost any field of activity. However, LEAN technologies should not be a onetime technique for optimizing any processes, but should become an integral element of the organization's management system.

The recognition of the need to apply an adequate strategy of change in the process of introducing lean manufacturing follows from two main prerequisites. The first of them is the analysis of the success / failure of the implementation processes, the second is the conviction that each case of implementation of lean manufacturing is unique and requires a largely individualized approach. Otherwise, changes will quickly become unusable, as there will be no infrastructure to continuously manage the process.

In addition, without assessing the organization's readiness for change, a project for the implementation of lean manufacturing is highly likely to cause contradictions with the corporate culture of the enterprise and will take place in a formal form, without bringing any benefit to the enterprise. Because, modern design tools offer engineers and technicians no choice but to standardize the working model.

For the successful implementation of this concept, a project office was created at JSC "Zlatmash", whose employees do a lot of work to involve all divisions of the enterprise in lean production. These include welding and coating areas, as well as assemblies of stationary and tabletop plates "Mechta" and "Zlata". The most important thing is that thanks to these actions, it is possible to improve the production process and increase the profit for a business entity. The most important thing is that thanks to these actions, it is possible to improve the production process and increase the profit for a business entity. However, it is necessary to 
focus on the needs of the operators. In our opinion, lean manufacturing should better explain the specific forms of work situations that allow you to adapt to modes of operation other than standard ones. Therefore, in the course of future activities, it is necessary to develop various scenarios for getting out of the current situations, the data about which are unknown at the time of project development.

\section{References}

1. J. R. X. Alves, J. M. Alves, Int. J. of Prod. Res., 53(17), 5320-5333 (2015)

2. S. Bhasin, P. Burcher., J. of Man. Tec. Man., 17(1), 56-72 (2006)

3. A. Pearce, D.Pons, Oper. Res. Persp. (2019)

4. J. P.Womack, D. T. Jones,. Lean thinking-banish waste and create wealth in your corporation. (J. of the Oper. Res. Soc., 1997)

5. K. Marley, P. Ward, Oper. Man. Res., 6 (1-2), 44-52 (2013)

6. M. F. van Assen, Oper. Man. Res., 11 (1-2) (2018)

7. P. Gaiardelli, B. Resta, S. Dotti, Intern. J. of LSS, 10 (1), 339-366 (2019)

8. N. Sinha, M. Matharu, J. of Ind. E. and M., 12 (2), 302-317 (2019)

9. P. Deflorin, M. Scherrer-Rathje, J. of Prod. Res., 50 (14), 3956-3973 (2012)

10. A. Panwar, B. P. Nepal, R. Jain, Prod. Plan. and C, 26 (7), 564-587 (2015)

11. T. Bortolotti, P. Romano, Prod. Plan. and C, 23 (7), 513-522 (2012)

12. A. Karim, K. Arif-Uz-Zaman, Bus. Proc. Man. J., 19 (1), 169-196 (2013)

13. N. A. Gorelov, V. V. Nikitina, Lab., Ec., 6 (4), 1285-1298 (2019)

14. O. B. Ivanov, ETAP, 1, 37-53 (2019)

15. A. T. Shilkina, Qual. Innov. Educ., 6, 49-57 (2020)

16. S. L. Poluektova, Bull. of the NCFU, 2 (71), 68-74 (2019) 